\title{
Search for Heavy Stable Charged Particle at the CMS experiment using the RPC Phase II upgrade
}

\author{
Junghwan Goh* on behalf of the CMS collaboration \\ Kyung Hee University, \\ 26 Kyungheedae-ro, Dongdaemun-gu, Seoul, 02447, Republic of Korea \\ E-mail: jhgohecern.ch
}

\begin{abstract}
Several theoretical models accommodate the possibility of Heavy Stable Charged Particles (HSCP). With improved data acquisition in the phase-II upgrade of the CMS-RPC system, triggering and identification of HSCPs are expected to be possible using the Time of Flight technique. Moreover, new RPC chambers will be installed to extend the acceptance up to $|\eta|<2.4$ with improved time and spatial resolution which can complement this search. Performance of new Level-1 trigger strategies to detect HSCPs at the High Luminosity LHC is presented.
\end{abstract}

The 39th International Conference on High Energy Physics (ICHEP2018)

4-11 July, 2018

Seoul, Korea

${ }^{*}$ Speaker. 
For the High Luminosity LHC (HL-LHC), the CMS experiment will upgrade several detectors which is known as Phase-II upgrade[1]. The phase-II upgrade of Resistive Plate Chamber (RPC) system involves new read out electronics (Link System), but also two layers of new chambers in the forward region at $1.9<|\eta|<2.4$ with improved detector technologies (iRPC).

The CMS RPC has an intrinsic time resolution less than 2ns which allows precise assignment of bunch crossing (BX) at $25 n s$ time window of currently installed link system. In the Phase-II upgrade, the new link system will provide improved timing information of $1 \sim 2$ ns resolution on top of delay due to signal propagation along readout strip in the chamber. For iRPCs, the delay will be corrected by using two electronics at both ends of strips.

In several theoretical models, Heavy Stable Charged Particle (HSCP) where its lifetime is long enough to be reconstructed as slowly moving muon-like trajectory in the CMS detector. HSCP can be discriminated from ordinary muon with their large time of flight (TOF).

The CMS RPC chambers are calibrated to give same timing for a particle produced at the origin moving close to the speed of light, such as muons from primary collision vertex. An HSCP at very low speed $(\beta=v / c \ll 1)$ will appear as a straight line with non-zero slope in distance-TOF plane. Therefore, a fast HSCP trigger can be designed based on least-squares fit of linear function of distance and TOF.
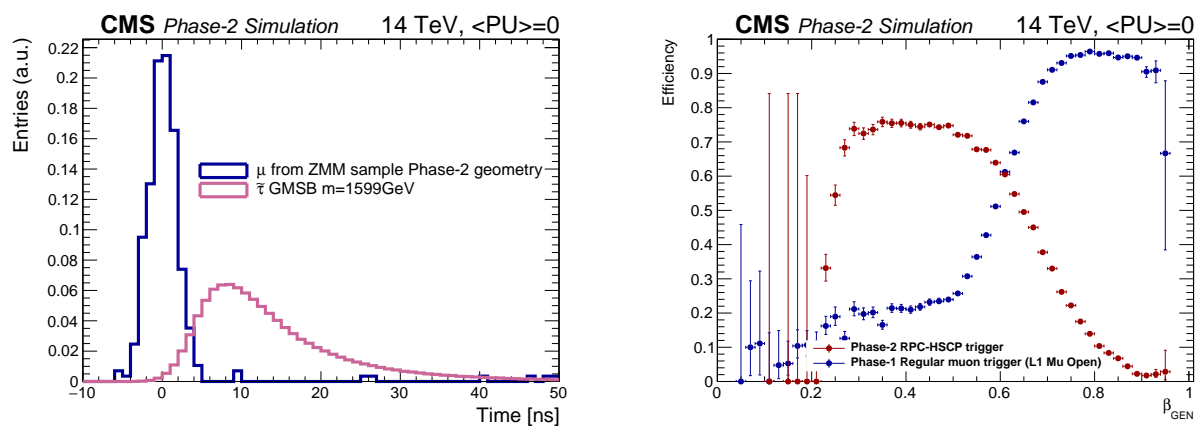

Figure 1: Left: Timing distributions of RPC hits from ordinary muons (blue) and HSCP (red) in the PhaseII upgrade. Right: Efficiency as a function of HSCP speed for the RPC-HSCP trigger (red) and the regular muon trigger (blue).

We measured performance of our implementation of HSCP trigger using Monte Carlo simulations. We choose supersymetry with stable $\tau$ pair as our benchmark model. The full CMS detector simulation and reconstruction in a Phase-II upgrade scenario is performed. For the ordinary muons, we used $Z^{0} / \gamma^{*} \rightarrow \mu^{+} \mu^{-}$process with the same setup.

As shown in the Fig. 1, the proposed HSCP trigger algorithm using the RPC timing improves trigger efficiency of HSCPs in $\beta<0.7$ at $90 \%$ which complements existing regular muon trigger. Therefore, upgrade of the RPC system can improve search for the HSCPs at the trigger level. We expect further improved with new developments including the iRPC.

\section{References}

[1] CMS Collaboration. The Phase-2 Upgrade of the CMS Muon Detectors. Technical Report CERN-LHCC-2017-012. CMS-TDR-016, CERN, Sep 2017. 